\title{
Citoyenneté et participation politique en Écosse. La décentralisation : une chance pour les femmes?
}

Citizenship and Political Participation in Scotland: Is Devolution an Opportunity for Women?

\section{Karine Rivière-De Franco}

\section{OpenEdition}

\section{Journals}

Édition électronique

URL : http://journals.openedition.org/rfcb/893

DOI : $10.4000 /$ rfcb. 893

ISSN : 2429-4373

Éditeur

CRECIB - Centre de recherche et d'études en civilisation britannique

Référence électronique

Karine Rivière-De Franco, « Citoyenneté et participation politique en Écosse. La décentralisation : une chance pour les femmes? », Revue Française de Civilisation Britannique [En ligne], XXI-1 | 2016, mis en ligne le 20 juillet 2016, consulté le 01 mai 2019. URL : http://journals.openedition.org/rfcb/893 ; DOI : $10.4000 /$ rfcb.893

Ce document a été généré automatiquement le 1 mai 2019.

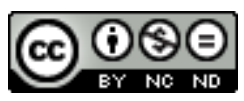

Revue française de civilisation britannique est mis à disposition selon les termes de la licence Creative Commons Attribution - Pas d'Utilisation Commerciale - Pas de Modification 4.0 International. 


\title{
Citoyenneté et participation politique en Écosse. La décentralisation : une chance pour les femmes?
}

\author{
Citizenship and Political Participation in Scotland: Is Devolution an Opportunity
} for Women?

Karine Rivière-De Franco

\section{Introduction}

1 Alors que les Écossaises, comme les Anglaises, durent attendre 1918 pour disposer du droit de voter et de siéger au Parlement de Westminster, établi depuis des siècles ${ }^{1}$, elles font partie intégrante du Parlement écossais depuis son (r)établissement en $1999^{2}$, à la fois comme électrices et comme représentantes. En théorie, la législation fait des femmes écossaises des citoyennes à part entière. En effet, l'une des trois formes de citoyenneté identifiées par le sociologue britannique Thomas Humphrey Marshall, la citoyenneté politique, relève du «droit de participer à l'exercice du pouvoir, comme membre d'une institution investie d'autorité politique ou comme électeur des membres de cette institution ${ }^{3}$. De même, la sociologue et politologue française Dominique Schnapper fonde la citoyenneté politique sur la participation politique, qui comprend le droit de vote, le droit d'éligibilité et le droit d'accéder à certaines fonctions publiques. ${ }^{4}$. Qu'en est-il, concrètement, de l'exercice de cette citoyenneté et de la participation effective des femmes à la vie politique écossaise? Dans quelle mesure le processus de dévolution a-t-il modifié les pratiques par rapport aux usages en cours à Westminster?

2 Cet article se propose d'étudier la notion de citoyenneté au prisme du processus de dévolution et de son impact sur la représentation et la participation politiques des femmes au Parlement écossais. Après avoir analysé les facteurs qui constituèrent un 
climat propice à une plus grande représentativité politique et leur impact sur la représentation descriptive de la population féminine lors des premières élections, l'action législative des femmes députées est examinée, au niveau de l'investissement quantitatif dans les activités parlementaires comme de l'intérêt témoigné pour les questions traditionnellement qualifiées d'« enjeux féminins ». Le corpus d'analyse permet de mettre en parallèle le comportement des élues au Parlement écossais avec celui de leurs consœurs à Westminster ; au sein de la chambre des Communes, les représentantes des circonscriptions écossaises sont considérées à part, afin d'apprécier le comportement législatif des Écossaises en fonction du lieu où elles siègent, Londres ou Édimbourg. Cette approche comparatiste vise à identifier d'éventuelles spécificités du Parlement écossais. La période d'étude choisie couvre les dix-huit premiers mois des législatures en place: de mai 2011 à décembre 2012 pour Édimbourg (Parlement dominé par le Scottish National Party) et de mai 2010 à décembre 2011 pour Westminster (Parlement dominé par la Coalition conservatrice-libérale démocrate).

\section{La représentation parlementaire des femmes écossaises}

\section{Un contexte propice à une meilleure représentativité}

3 À l'aube du premier scrutin pour le Parlement écossais de 1999, plusieurs facteurs semblaient contribuer à créer un climat favorable à une meilleure représentativité politique par rapport à la Chambre des communes de Westminster. Tout d'abord, la nouveauté même de l'institution pouvait laisser espérer de futurs élus qui refléteraient davantage la population en termes de caractéristiques sexuées. Comme le souligne l'universitaire britannique Carmel Roulston :

[L]a plupart des constitutions ont été établies par des hommes et sont le reflet d'une vision masculine du pouvoir, de la politique et de la citoyenneté; exclus du processus de réflexion autour de ces constitutions, les intérêts des femmes ont fréquemment été négligés 5 .

Dans le cas de la décentralisation politique en Écosse, les femmes ont participé activement aux débats sur la création du nouveau Parlement et ont été en mesure d'influencer son fonctionnement, ainsi que le mode de scrutin ou de sélection des candidats. Comme en atteste Mike Watson - qui fut député à Westminster et à Holyrood la Chambre des communes de Londres constituait un contre-modèle, l'exemple même des pratiques qu'il convenait de ne pas reproduire ${ }^{6}$. Il s'agissait, au contraire, d'engager un renouveau démocratique, de créer une institution à l'atmosphère plus consensuelle, plus informelle, avec des horaires davantage compatibles avec une vie de famille et un équilibre politique différent, au sein duquel les questions d'égalité occuperaient une position centrale. C'est ainsi que, parmi les quatre recommandations du Constitutional Steering Group ${ }^{7}$, qui furent ensuite adoptées par le Parlement, figure l'égalité des chances. De même, un comité parlementaire permanent travaille sur ce thème, un groupe en charge de la promotion de l'égalité a été créé au sein du pouvoir exécutif, et une femme doit occuper le poste de président ou de vice-président du Parlement. Par ailleurs, le débat sur la dévolution a coïncidé avec une évolution au sein des mouvements féministes ; à partir des années 1980, leur action s'est institutionnalisée et les femmes se sont mises à militer pour le changement de l'intérieur en investissant syndicats, groupes 
de pression, partis et institutions politiques ${ }^{8}$. Comme l'explique Nathalie Duclos dans son ouvrage Femmes, Pouvoir et Nation en Écosse, «les autonomistes et les féministes des années 1980 et 1990 purent se rejoindre dans le rejet du modèle politique traditionnel $»^{9}$. Si les nationalistes ont fondé une partie de leur argumentation sur le concept du "déficit démocratique » dont souffrait l'Écosse, les femmes s'en saisirent pour démontrer qu'elles étaient victimes d'un « double déficit démocratique ». Dès lors, «voter 'oui' [lors du référendum de 1997 sur la création d'un Parlement écossais] n'était pas seulement un choix nationaliste mais également un choix féministe et le référendum une occasion à ne pas manquer ${ }^{10}$, et les organisations féministes parvinrent à organiser une mobilisation importante. Le mode de scrutin adopté, qui introduisait une dose de proportionnelle, à la différence du système pratiqué pour Westminster, était également, en principe, favorable à une plus grande diversité. Le mode de scrutin mixte (Additional Member System, AMS) prévoyait que chaque électeur disposât de deux votes: un pour élire le candidat de sa circonscription (Member of the Scottish Parliament, MSP) à travers un système majoritaire (First-Past-The-Post, comme pour Westminster) et un second pour choisir des membres régionaux à partir d'un scrutin de liste. Ce second vote avait pour objectif de corriger les excès du système majoritaire dans les circonscriptions et d'introduire une dose de proportionnalité. Or, une forte corrélation a pu être observée entre la représentation proportionnelle et un nombre plus élevé de femmes élues ${ }^{11}$. Enfin, la représentation des femmes a également été facilitée par l'absence de députés en place (incumbents). Pour reprendre la typologie, établie par Pippa Norris et Joni Lovenduski, qui décrit les quatre types de facteurs qui influencent la participation politique des femmes - les facteurs systémiques, les facteurs internes aux partis, les facteurs individuels et les élections elles-mêmes - un certain nombre des obstacles à la représentation féminine semblaient levés en Écosse ${ }^{12}$. En termes de représentation numérique, également qualifiée de représentation descriptive, représentation miroir ou représentation de groupe, les élections écossaises se sont-elles effectivement révélées significatives pour la population féminine écossaise, comme les circonstances pouvaient le laisser penser?

\section{Une représentation descriptive substantielle}

5 À l'issue du premier scrutin en 1999, les femmes constituaient près de $40 \%$ des membres du Parlement écossais (37\%), un chiffre qui démontre clairement les bénéfices de la dévolution en termes de représentation numérique. En effet, à la même période, les élues à Westminster étaient deux fois moins nombreuses (18,2 \%, après le scrutin de 1997 au terme duquel la représentation féminine doubla quasiment par rapport à 1992). Conformément aux espoirs placés dans le nouveau Parlement, cette première élection constitua un réel succès pour les femmes écossaises. Toutefois, l'évolution lors des consultations électorales suivantes s'est avérée décevante, puisque, à l'exception de la deuxième élection, les chiffres sont restés inférieurs à ceux de 1999 (39,5\% en 2003, 33 \% en 2007, $35 \%$ en 2011). Comme le souligne le groupe Engender - qui milite pour améliorer la condition des femmes en Écosse - depuis sa création, le Parlement écossais est passé de la $4^{\mathrm{e}}$ à la $13^{\mathrm{e}}$ place dans le classement international de la représentation parlementaire des femmes ${ }^{13}$. Par ailleurs, contrairement aux attentes engendrées par l'introduction d'une dose de proportionnelle dans le mode de scrutin, l'analyse affinée des résultats électoraux révèle que la majorité des candidates n'a pas été élue grâce aux listes régionales, mais dans les circonscriptions (avec le système majoritaire utilisé pour Westminster): elles représentaient $41 \%$ des députés dans les circonscriptions, contre 
$32 \%$ des représentants régionaux ${ }^{14}$. Les bons résultats en termes de représentation féminine n'étaient donc pas dus au système électoral, mais aux mesures adoptées par certains partis politiques lors de leur procédure de sélection des candidats. Le parti travailliste avait adopté le principe du jumelage de circonscriptions limitrophes dans lesquelles un homme et une femme étaient nominés (twinning), d'où un groupe parlementaire composé à $50 \%$ de femmes, tandis que le Scottish National Party avait, lui, choisi de placer les candidates en tête sur ses listes régionales, avec comme résultat $42,8 \%$ de femmes au Parlement ${ }^{15}$. Le manque d'engagement en la matière des conservateurs et des libéraux-démocrates s'était reflété dans le nombre peu élevé de leurs représentantes (16,7 et 11,8 \% de leur groupe parlementaire respectif). En Écosse, comme à Westminster, les partis agissent comme des "gatekeepers ${ }^{16}$, et seules "les mesures qui garantissent l'égalité» s'avèrent efficaces, la rhétorique d'égalité (les déclarations de bonnes intentions) et la promotion de l'égalité (l'accompagnement des candidates) se révèlent insuffisantes ${ }^{17}$.

6 Sur la période d'étude choisie - mai 2011 à décembre 2012 pour Édimbourg et mai 2010 à décembre 2011 pour Westminster - les femmes représentaient plus d'un tiers des députés à Édimbourg (35\%), contre moins d'un quart à Westminster (22\%). Quant aux élues de Westminster représentant des circonscriptions écossaises, elles constituaient $22,3 \%$ de l'ensemble des parlementaires des Communes. Par ailleurs, en Écosse, le parti travailliste disposait du plus grand nombre d'élues ( $46 \%$ ), suivi par les conservateurs ( $40 \%$ ), le SNP (24,6\%) et les libéraux-démocrates ( $20 \%$ ). Enfin, sur la période, l'Écosse a connu plusieurs femmes à la tête de partis politiques - Johann Lamont, leader des travaillistes et Ruth Davidson, chef des conservateurs - une situation qui contraste avec celle des partis basés à Londres ${ }^{18}$. Le poids respectif des députées au sein de leur Parlement a-t-il un impact sur l'investissement de ces élues, tant sur le plan quantitatif - leur degré de participation à la vie parlementaire - que qualitatif - le type d'enjeux pour lesquels elles se mobilisent?

\section{L'action législative des députées écossaises}

\section{La contribution globale des députées écossaises au travail législatif}

$7 \quad$ La grille d'activités parlementaires qui a été établie offre à la fois une vision globale - à travers l'ensemble des contributions écrites et orales - ainsi qu'une perspective plus détaillée - séances de questions au Premier ministre, projets de loi, motions ou votes. Considérant l'activité globale des députées, c'est-à-dire l'ensemble de leurs interventions orales et écrites au sein du Parlement écossais ${ }^{19}$, les femmes sont à l'origine de près de la moitié $(45,2 \%)$ des contributions (5385 contre 6532 pour les hommes), une surparticipation de 10 points par rapport à leur représentation parlementaire (voir le tableau $\mathrm{n}^{\circ}$ 1). À Westminster, les femmes députés n'y contribuent qu'à hauteur de 19,4\% (32 131 interventions contre 133533 pour les hommes), soit une légère sous-participation (-2,6 points). Les parlementaires en Écosse se montrent beaucoup plus actives que leurs collègues de Westminster; quant au comportement législatif des élues qui représentent des circonscriptions écossaises à la Chambre des communes, il se situe entre celui des deux groupes à $25.6 \%$ (2 352 interventions contre 9172 pour les hommes), avec une légère sur-participation $(+3,3$ points). 
Tableau $n^{\circ} 1$ : La contribution globale des femmes députés au travail législatif

(Holyrood mai 2011-décembre 2012, Westminster, mai 2010-décembre 2011, \% / points)

\begin{tabular}{|l|l|}
\hline Holyrood & $45,2(+10,2)$ \\
\hline Westminster & $19,4(-2,6)$ \\
\hline Circonscriptions écossaises, Westminster & $25,6(+3,3)$ \\
\hline
\end{tabular}

Source : I'auteur, calculé d'après Official Report (Parlement écossais), Hansard Parliamentary Archives (Parlement de Westminster).

\section{La contribution des députées écossaises aux activités parlementaires spécifiques}

9 Les Parlements de Holyrood et de Westminster présentent des activités comparables qui permettent d'évaluer l'activité de leurs membres par rapport à leur représentation descriptive respective. Ainsi, tous deux disposent de séances de questions au Premier ministre. À la Chambre des communes de Londres, le président, John Bercow, a adressé une lettre aux leaders des trois principaux partis au mois de février 2014 dans l'optique de réformer cette demi-heure hebdomadaire, qu'il juge "sexiste, snob et dévoyée " ${ }^{20}$. En Écosse, contrairement à ce qui avait été préconisé lors des discussions préparatoires, cette pratique a été adoptée sous une forme quasi similaire ${ }^{21}$. Sur l'ensemble de la période d'étude, les questions posées par des femmes lors des séances de questions au Premier ministre écossais représentent près de la moitié de l'ensemble des questions (47,6 \%, 571 interventions contre 629 pour leurs homologues masculins) (voir le tableau $\mathrm{n}^{\circ} 2$ ). $\mathrm{Ce}$ pourcentage s'avère supérieur à leur représentation numérique de plus de 12 points. Les chiffres équivalents pour Westminster montrent, eux, un investissement dans cette activité pratiquement à la hauteur de la représentation descriptive des députées $(21,8 \%$, $-0,2$ points). Quant aux interventions des femmes qui représentent des circonscriptions écossaises, elles se révèlent légèrement supérieures à leur représentation $(26,8 \%,+4,5$ points). De grandes différences apparaissent donc selon le groupe considéré, avec, d'une part, une plus grande activité des élues au Parlement écossais et, d'autre part, au sein de Westminster, des contributions plus nombreuses de la part de celles qui représentent des circonscriptions écossaises.

Les deux législatures offrent la possibilité de déposer des motions; ces dernières permettent aux députés d'exprimer leur point de vue sur un sujet particulier ou d'initier un débat et d'obtenir le soutien de leurs collègues, qui peuvent y apposer leur signature, comme pour une pétition. En Écosse, près d'un tiers de ces motions a été rédigé par des femmes (30,2\%, 1624 contre 69 pour les hommes), ce qui représente une sousparticipation comparée à leur présence parlementaire $(-4,8$ points). À Westminster, les motions dont les femmes sont à l'origine constituent $16 \%$ du nombre total (410 pour les femmes, 2152 pour les hommes, -6 points). Sans être totalement négligées, au sein des deux Parlements, les motions ne représentent pas une activité dans laquelle les femmes s'investissent de manière importante. Il est vrai que les motions n'apportent pas de résultat concret immédiat, ce qui explique que l'ancien Premier ministre John Major les 
ait qualifiées d' «équivalent parlementaire $d u$ graffiti ${ }^{22}$. En revanche, les députées écossaises à Westminster adoptent une attitude très différente : il s'agit pour elles d'une activité très prisée $(44,2 \%,+21,9$ points), qui est d'ailleurs moins soumise à la stricte discipline de parti que d'autres travaux.

Le Parlement écossais - dont les pouvoirs ont été définis par le Scotland Act de 1998 puis étendus par le Scotland Act de 2012 - peut légiférer dans les domaines qui relèvent de la décentralisation, comme l'agriculture, l'éducation, l'environnement, les services de santé et les services sociaux, le logement, le gouvernement local, les sports et les arts, le tourisme ou les transports ${ }^{23}$. En matière de dépôt de projets de loi, les élues adoptent un comportement similaire à Holyrood et à Westminster. Elles y participent légèrement moins que ce que leur influence numérique pourrait laisser penser: une sousparticipation de 4,2 points est observée en Écosse (30,8 \%) et de 2,5 points aux Communes $(19,5 \%)$. Les représentantes des circonscriptions écossaises à Westminster se distinguent, elles, par l'absence totale de dépôt de projet de loi (-22,3 points).

Tableau $n^{\circ} 2:$ Les activités parlementaires des femmes députés

(Holyrood mai 2011-décembre 2012, Westminster, mai 2010-décembre 2011, \% / points)

\begin{tabular}{|l|l|l|l|}
\hline & Holyrood & Westminster & $\begin{array}{l}\text { Circonscriptions } \\
\text { Westminster }\end{array}$ \\
\hline $\begin{array}{l}\text { Questions au Premier } \\
\text { ministre }\end{array}$ & $\begin{array}{l}47,6 \\
12,6)\end{array}$ & $21,8(-0,2)$ & $26,8(+4,5)$ \\
\hline Projets de loi & $30,8(-4,2)$ & $19,5(-2,5)$ & $0(-22,3)$ \\
\hline Motions & $30,2(-4,8)$ & $16(-6)$ & $44,2(+21,9)$ \\
\hline
\end{tabular}

Source : l'auteur, calculé d'après Official Report (Parlement écossais), Hansard Parliamentary Archives (Parlement de Westminster).

13 Le comportement de vote représente un autre indicateur de l'activité législative, qui comporte deux éléments : la fréquence de vote et le respect ou non de la ligne de parti, qui peut être estimé à partir du nombre de votes dits de « rébellion ». À Holyrood, les taux de participation au vote des femmes et des hommes s'avèrent très proches $(94,1 \%$, contre $94,3 \%$ ) (tableau n 3), à l'instar de ce qui est observé à Westminster (72,5\% contre 72,3\%). En revanche, au sein des députés qui représentent des circonscriptions écossaises, l'écart s'avère plus notable, les femmes votant plus fréquemment que leurs collègues masculins $(69,5 \%$ contre $63,4 \%,+6,1$ points). Au niveau du respect ou de la défiance par rapport à la discipline de vote, les taux de rébellion restent très faibles, toutefois les conduites diffèrent nettement : alors que le taux de rébellion pour les femmes MSPs est supérieur à celui de leurs collègues masculins $(0,3 \%$ contre $0,2 \%)$, le phénomène inverse se produit à Westminster (0,5\% contre 1,3\%). En Écosse, les élues affirment davantage leur liberté d'action et font preuve d'une plus grande indépendance qu'à Westminster, où la discipline de vote prévaut, que ce soit par loyauté ou par crainte d'être stigmatisées. Le taux de rébellion des députées qui représentent des circonscriptions écossaises est également inférieur à celui des hommes ( $0,2 \%$ contre $0,7 \%)$, ces femmes se conformant au modèle de comportement majoritaire à Westminster. 
Tableau $\mathrm{n}^{\circ} 3$ : Le vote des femmes au Parlement, participation et « rébellion »

\begin{tabular}{|l|l|l|l|}
\hline \multirow{4}{*}{ Holyrood } & & Participation & Rébellion \\
\hline & Femmes & 94,1 & 0,3 \\
\cline { 2 - 4 } & Hommes & 94,3 & 0,2 \\
\cline { 2 - 4 } & Ecart F / H & $-0,2$ & 0,1 \\
\hline \multirow{5}{*}{ Westminster } & Femmes & 72,5 & 0,5 \\
\cline { 2 - 4 } & Hommes & 72,3 & 1,3 \\
\cline { 2 - 5 } & Ecart F / H & 0,2 & $-0,8$ \\
\hline \multirow{4}{*}{ Circonscriptions écossaises, Westminster } & Hommes & 63,4 & 0,7 \\
\cline { 2 - 5 } & Femmes & 69,5 & 0,2 \\
\cline { 2 - 5 } & Ecart F / H & 6,1 & $-0,5$ \\
\hline
\end{tabular}

Source : l'auteur, calculé à partir des chiffres relevés par The Public Whip.

15 En Écosse, dans le cadre d'une représentation descriptive supérieure, les élues s'investissent davantage dans le travail législatif ; leur sur-participation globale, ainsi que leurs nombreuses interventions lors des questions au Premier ministre, tranchent avec la sous-participation des députées de Westminster. Le nombre de représentantes, l'atmosphère de travail, et les attentes créées par cette nouvelle institution en matière d'égalité, s'avèrent propices à la participation parlementaire des femmes. Pour ce qui est des représentantes de circonscriptions écossaises à Westminster, elles contribuent globalement davantage aux travaux de la Chambre que les autres députées de Westminster ; en revanche, leur activité est inférieure à celle des députées du Parlement écossais. Quel que soit leur degré d'investissement, l'action des députées s'avère-t-elle bénéfique pour l'ensemble de la population féminine?

\section{L'engagement des députées écossaises pour les " enjeux féminins »}

Dès sa création, Holyrood a assuré un meilleur équilibre que Westminster entre les représentants des deux sexes. La représentation numérique s'avère d'autant plus importante qu'une corrélation existerait entre la représentation descriptive et la représentation substantive: les intérêts spécifiques de la population féminine seraient ainsi mieux pris en compte par les femmes politiques que par les hommes. Une telle hypothèse repose, en partie, sur la notion de "masse critique ", le principe selon lequel lorsque les femmes atteignent un certain seuil numérique - entre 15 et $40 \%$ de l'ensemble 
des parlementaires - elles sont alors en mesure d'influencer la culture et le discours politiques dominants ainsi que l'agenda politique ${ }^{24}$. Dans le cadre de la décentralisation britannique, une représentation parlementaire numérique supérieure garantit-elle, effectivement, aux femmes écossaises une meilleure prise en compte des «enjeux féminins »? Les études menées jusqu'à présent ont porté, pour la plupart, sur les députées travaillistes des années 1990 à Westminster, avec les travaux de Pippa Norris, Joni Lovenduski, Sarah Childs, Karen Bird ou Rosie Campbell ${ }^{25}$. Elles ont permis d'identifier de légères variations au niveau des valeurs, des comportements et de l'implication dans les politiques sociales, mais ont conclu que le meilleur indicateur du comportement parlementaire reste l'affiliation politique. Dans les années 1970, Michael J. Keating, a, lui, montré que les députés qui représentaient des circonscriptions écossaises à Westminster concentraient leur action sur des enjeux spécifiquement écossais ${ }^{26}$. Les recherches effectuées sur les premières années du Parlement écossais, conduites par Paul Chaney et Sylvia Shaw, ont révélé l'influence des femmes sur la "politique postdévolution »; ils ont ainsi établi que les élues participaient à tous les types de débats et qu'elles avaient davantage soulevé la question de la violence faite aux femmes que les hommes ${ }^{27}$. Qu'en est-il sur notre période d'étude?

\section{La part des « enjeux féminins » dans l'action législative des députées écossaises}

17 Pour l'étude, les « enjeux féminins » ont été identifiés grâce aux définitions établies par Joni Lovenduski et Azza Karam d'une part, Linda Trimble et Jane Arscott, d'autre part. Pour les premières, il s'agit de questions qui concernent en premier lieu les femmes, que ce soit pour des raisons biologiques (dépistage du cancer du sein ou contraception) ou pour des motifs sociaux (parité ou garde d'enfants); les secondes font référence aux politiques dont les répercussions directes et immédiates touchent davantage les femmes que les hommes ${ }^{28}$. Les députées au Parlement écossais se distinguent-elles par un engagement particulier pour ce type de sujets?

18 L'analyse du contenu des interventions des élues révèle que les «enjeux féminins » se situent davantage au cœur des préoccupations des femmes aux Communes qu'à Holyrood. Ainsi, les députées écossaises posent quatre fois moins de questions sur ces thématiques à leur Premier ministre Alex Salmond (sur la santé des femmes, la journée internationale des femmes, la violence conjugale ou l'accueil des jeunes enfants) que leurs consœurs de Westminster à David Cameron (sur l'égalité au travail, les allocations, les retraites, la santé et la violence conjugale) (2,1\% contre $8 \%$ ) (tableau $\left.n^{\circ} 5\right)$. Parmi les motions déposées par les femmes parlementaires, le même phénomène apparaît, avec une implication moindre des élues écossaises : 4,9\% des motions dont elles sont à l'origine portent sur des « enjeux féminins » contre 6,1\% à Westminster. Ces motions soulèvent des problématiques liées à la violence conjugale, à l'emploi, à la pauvreté, aux services de maternité, à l'avortement, à l'égalité salariale ou à la retraite. En revanche, en Écosse, l'un des quatre projets de loi proposés par des femmes concerne directement la population féminine : il vise à lutter contre la prostitution (Proposed criminalisation of the purchase of sex (Scotland) bill). À Westminster, l'unique projet qui relève des "enjeux féminins" propose de garantir l'anonymat aux personnes accusées de viol (Anonymity (Arrested Persons) Bill) ; il représente 2,4 \% de l'ensemble des projets de loi déposés par les députées. En matière de votes de rébellion, moins de $5 \%$ sont motivés par des « enjeux féminins », à 
Édimbourg $(4,9 \%)$ comme à Westminster $(4,3 \%)$. Les femmes décident de se démarquer de la ligne officielle prônée par leur parti sur d'autres types de problématiques, ce qui s'avère d'autant plus étonnant en Écosse où le taux de rébellion des femmes est plus élevé.

Ces résultats peuvent paraître surprenants et ils ne semblent pas, à première vue, renforcer la thèse de la corrélation entre les deux types de représentation. Alors que les élues écossaises bénéficient d'une représentation numérique supérieure et qu'elles contribuent davantage au travail législatif, les thèmes sur lesquels elles choisissent de travailler affectent moins directement la population féminine que l'action législative des élues, pourtant moins nombreuses, de Westminster. Par ailleurs, quel que soit le Parlement, les «enjeux féminins» ne constituent qu'une minorité de l'action parlementaire des députées, qui ne peuvent donc pas être considérées comme des porteparole de la population féminine. L'activité politique des députées en Écosse peut être considérée comme décevante par les militants pour l'égalité et les défenseurs de la théorie liant représentations descriptive et substantive. Il convient toutefois de relativiser cet apparent manque d'implication, qui ne signifie pas forcément que les intérêts des femmes écossaises sont ignorés par la classe politique. En effet, de par ses origines, le Parlement écossais s'avère sensible aux questions d'égalité et ces thématiques ont été intégrées à la manière de gouverner et de légiférer. L'ensemble des parlementaires et du pouvoir exécutif doit donc en tenir compte. Ainsi, la question de la violence conjugale a, par exemple, été au cœur du premier Parlement écossais; le gouvernement a fait adopter son projet de loi sur les agressions sexuelles (Sexual Offences Bill) et une commission a déposé un autre projet, également voté par le Parlement, sur la protection contre la violence (Protection from Abuse Bill) ${ }^{29}$. À Westminster, le positionnement des femmes qui représentent les circonscriptions écossaises diffère grandement selon l'activité considérée. Alors que pour les questions au Premier ministre et les motions, ces élues affichent un intérêt certain pour les « enjeux féminins ", posant par exemple sept fois plus de questions au Premier ministre sur ces sujets que les députées en Écosse et deux fois plus que le reste des députées de Westminster, aucun de leurs votes de rébellion ne porte sur ces problématiques. Malgré ce relatif désintérêt pour les « enjeux féminins », qui sont les députées qui choisissent de s'en préoccuper?

Tableau $n^{\circ} 5$ : La part des « enjeux féminins » dans l'activité législative des députées

(Holyrood mai 2011-décembre 2012, Westminster, mai 2010-décembre 2011, \%)

\begin{tabular}{|l|l|l|l|l|}
\hline & $\begin{array}{l}\text { Questions au Premier } \\
\text { ministre }\end{array}$ & Motions & $\begin{array}{l}\text { Projets de } \\
\text { loi }\end{array}$ & $\begin{array}{l}\text { Votes de } \\
\text { rébellion }\end{array}$ \\
\hline Holyrood & 2,1 & 4,9 & 25 & 4,9 \\
\hline Westminster & 8 & 6,1 & 2,4 & 4,3 \\
\hline $\begin{array}{l}\text { Circonscriptions écossaises, } \\
\text { Westminster }\end{array}$ & 15,8 & 8,7 & 0 & 0 \\
\hline
\end{tabular}

Source : l'auteur, calculé d'après Official Report (Parlement écossais), Hansard Parliamentary Archives (Parlement de Westminster). 


\section{L'engagement individuel des députées écossaises pour les « enjeux féminins "}

21 Au-delà des effets théoriques de la représentation numérique, certaines études - comme celles de Paul Chaney ou Suzanne Dovi ${ }^{30}$ - ont souligné le rôle crucial joué par des députées activistes et militantes, qualifiées d' "equality champions", des personnes qui font preuve d'un engagement fort en matière d'égalité. Il convient d'analyser le travail des femmes parlementaires dans le détail, afin d'identifier l'existence ou non de ces députées, ce qui permet par là même d'évaluer l'engagement respectif des différents partis politiques en matière d'égalité. Les chiffres révélant un relatif désintérêt global pour les enjeux féminins reflètent-ils une tendance uniforme chez les députées ou cachent-ils d'importantes différences de comportement?

Au Parlement écossais, plus de $70 \%$ de l'ensemble des questions orales et écrites qui portent sur des « enjeux féminins » ont été posées par seulement cinq femmes, dont $30 \%$ par Jackie Baillie et $12 \%$ par Helen Eadie, deux membres du parti travailliste. D'ailleurs, près de $80 \%$ de l'ensemble de ces questions émanent de représentantes travaillistes (Elaine Smith, Jackie Baillie, Helen Eadie, Jenny Marra, Kezia Dugdale, Margaret McDougall, Patricia Ferguson, Sarah Boyack, Anne McTaggart). De même, plus d'un tiers des motions sont le fait de seulement cinq députées : Jackie Baillie, Elaine Smith, Mary Fee, Christina McKelvie et Alison Johnston, les trois premières appartenant au Parti travailliste. En outre, la moitié des questions au Premier ministre a été posée par Johann Lamont, élue travailliste, et, au total, $75 \%$ des questions proviennent du parti travailliste (Elaine Smith, Jenny Mara, Jackie Baillie). Enfin, le projet de loi sur la prostitution a été déposé par une autre députée travailliste, Rhoda Grant. Au sein du Parlement écossais, toutes les élues sont donc loin de s'engager pour les « enjeux féminins ", qui sont portés par un nombre relativement limité de députées - en particulier Jackie Baillie, Johann Lamont et Elaine Smith -, essentiellement issues du parti travailliste.

Cette importance disproportionnée d'un petit groupe de parlementaires en Écosse se retrouve-t-elle à Westminster ou s'avère-t-elle spécifique à l'institution décentralisée ? Aux Communes, plus de $70 \%$ des motions ont été déposées par des femmes différentes, dont la moitié par des députées travaillistes (Theresa Pearce, Katy Clark, Harriet Harman, Glenda Jackson, Alison McGovern, Valerie Vaz), même si des députées libéralesdémocrates y ont également participé (Burt Lorely, Jo Swinson). Quasiment toutes les questions au Premier ministre (15 sur 16) émanent de femmes différentes, majoritairement issues du parti travailliste (Valerie Vaz, Sarah Hodgson, Jessica Morden, Rachel Reeves, Jenny Chapman, Gloria De Piero, Pamela Nash, Fiona O'Donnell pour le parti travailliste, Helen Grant, Anna Soubry, Mary Macleod et Harriett Baldwin pour le parti conservateur, Jo Swinson et Annette Brooke pour les libérales-démocrates). Enfin, le projet de loi a été déposé par une députée conservatrice, Anna Soubry. Contrairement au Parlement écossais, l'analyse détaillée de l'activité des parlementaires de Westminster en matière d' " enjeux féminins » ne fait pas émerger d' « equality champions »; le Parlement de Westminster ne dispose pas d'un noyau dur de femmes qui interviennent fréquemment sur ces sujets. Pour reprendre les termes de Paul Chaney, il existe bien, au sein de l'institution écossaise, des «individus clés qui interviennent de manière répétée pour faire avancer les questions féministes et d'égalité des sexes $"^{31}$. Par ailleurs, le parti travailliste ressort nettement comme le plus engagé sur ces thématiques, même si la tendance est 
moins marquée à Westminster. Concernant les représentantes des circonscriptions écossaises à Westminster, les trois motions qui portent sur des " enjeux féminins » ont été rédigées par deux femmes: Katy Clark (travailliste) et Jo Swinson (libéraledémocrate), tandis que Pamela Nash, Fiona O'Donnell (travaillistes) et Jo Swinson (libérale-démocrate) ont chacune interpellé le Premier ministre à une reprise. Au sein de ce groupe d'élues, peu se montrent intéressées par les «enjeux féminins », et celles qui les abordent ne le font que de manière très ponctuelle, la libérale-démocrate Jo Swinson apparaissant comme la plus active, avec trois interventions en 18 mois.

\section{Conclusion} citoyenneté politique dans le contexte de la décentralisation en Écosse. L'analyse a révélé que l'hypothétique corrélation entre représentation numérique, investissement quantitatif et défense des " enjeux féminins » s'avère en réalité beaucoup plus complexe. Le Parlement écossais présente la spécificité de bénéficier du travail d'un petit nombre de députées fortement mobilisées sur les "enjeux féminins ", et, comme à Westminster, les élues travaillistes apparaissent comme les plus engagées sur ces questions. En revanche, si un nombre plus élevé de femmes députées entraîne, dans le cas de l'Écosse, un investissement plus important en termes quantitatifs, il ne signifie pas automatiquement un intérêt plus marqué pour les questions susceptibles de toucher les femmes ${ }^{32}$. Toutefois, l'action des premiers gouvernements écossais atteste d'une réelle préoccupation pour ces questions. Depuis 1999, le Parlement a ainsi légiféré - parfois à plusieurs reprises - sur les questions d'agressions sexuelles et de viol, de violences conjugales, d'allaitement, de prostitution ou de mariages forcés ${ }^{33}$. Il semble donc que les femmes politiques, comme les activistes au sein des associations, aient réussi à influencer la création de cette nouvelle institution, qui se montre plus soucieuse des questions d'égalité que le Parlement de Westminster, ce qui constitue un véritable progrès. Même si l'action des femmes députées, la définition des « enjeux féminins » et le concept de démocratie représentative peuvent être influencés par les théories sur l'intersectionnalité, il n'en demeure pas moins, qu'en se fondant sur les principes d'équité et de justice ${ }^{34}$, la décentralisation politique s'est avérée positive pour les femmes écossaises. La grande hétérogénéité des positions des partis politiques en matière de parité et d'égalité reflète, quant à elle, l'absence de contraintes légales qui s'appliqueraient à tous, d'où la conclusion de Joyce McMillan et Ruth Fox dans leur rapport sur la dévolution rédigé pour la Hansard Society: la nécessité à l'avenir d'aller au-delà des mesures adoptées de manière volontaire par certains partis ${ }^{35}$.

Karine Rivière-De Franco est maître de conférences en civilisation britannique à l'Université d'Orléans et membre du CEPOC (Centre d'Études Politiques C ontemporaines), composante de POLEN (EA 4710). Elle travaille sur la communication politique, les médias et les femmes politiques en Grande-Bretagne. Elle est l'auteur de La communication électorale en Grande-Bretagne. De M. Thatcher à T. Blair (L'Harmattan, 2008), ainsi que de nombreux articles ; elle a co-dirigé deux ouvrages sur l'image, la communication et les stratégies électorales et a dirigé un numéro de La Revue LISA sur l'égalité des droits.

Revue Française de Civilisation Britannique, XXI-1 | 2016 


\section{BIBLIOGRAPHIE}

Auer, Christian, Dubois-Nayt, Armel \& Duclos, Nathalie, Femmes, Pouvoir et Nation en Écosse du XVIe siècle à aujourd'hui (Villeneuve d'Ascq, Presses du Septentrion, 2012).

Bennie, Lucie, Denver, David, Mitchell, James \& Bradbury, Jonathan, "Harbingers of New Politics? The Characteristics and Attitudes of Candidates in the Scottish Parliamentary Election 1999", British Elections and Parties 11 (2001), pp. 23-45.

Breitenbach, Esther \& Mackay, Fiona, Women and Contemporary Scottish Politics. An Anthology (Édimbourg, Polygon at Edinburgh, 2001).

Bromley, Catherine, Curtice, John, McCrone, David \& Park, Alison (eds), Has Devolution Delivered? (Édimbourg, Edinburgh University Press, 2006).

Brown, Alice, "Designing the Scottish Parliament", Parliamentary Affairs 53:3 (July 2007), pp. 542-556.

Brown, Alice, “Representing Women in Scotland”, Parliamentary Affairs 51:3 (July 1998), pp. 435-444.

Cavanagh, M., Shapard, M. \& McGarvey, N, "New Scottish Parliament, New Scottish Parliamentarians?", Journal of Legislative Studies (September 2000), pp. 79-104.

Chaney, Paul, Mackay, Fiona \& McAllister, Laura, Women, Politics and Constitutional Change (Cardiff, University of Wales Press, 2007).

Childs, Sarah, Women and British Party Politics: Descriptive, Substantive and Symbolic Representation (Londres, Taylor and Francis, 2008).

Childs, Sarah, Lovenduski, Joni \& Campbell, Rosie, Women at the Top 2005. Changing Numbers, Changing Politics? (Londres, Hansard Society, 2005).

Dovi, Suzanne, The Good Representative (Oxford, Blackwell, 2007).

Hassan, Gerry \& Warhurst, Christ (eds), The New Scottish Politics. The First Year of the Scottish Parliament and Beyond (Londres, Stationary Office, 2000).

Hough, Dan \& Jeffery, Charlie (eds), Devolution and Electoral Politics (Manchester, Manchester University Press, 2006).

Karam, Azza, Women in Parliament: Beyond Numbers (Stockholm, IDEA, 1998).

Keating, Michael, A Test of Integration: the Scottish Members of Parliament (Glasgow, Centre for the Study of Public Policy, University of Strathclyde, 1977).

Lovenduski, Joni, Feminizing Politics (Cambridge, Polity Press, 2005).

Lovenduski, Joni (ed.), State Feminism and Political Representation (Cambridge, Cambridge University Press, 2005).

McFadden, Jean \& Lazarowitz, Mark, The Scottish Parliament. An Introduction (Haywards Heath, Bloomsbury Professional, 2010). 
Molinari, Véronique, « La campagne dans la campagne : pour la parité au sein des nouvelles assemblées autonomes ", Revue française de civilisation britannique 14:1 (« La dévolution des pouvoirs à l'Écosse et au Pays de Galles ») (2006), pp. 165-176.

Norris, P., Vallance, E. \& Lovenduski, J., "Do Candidates Make a Difference? Gender, Race, Ideology and Incumbency”, Parliamentary Affairs 45:4 (octobre 1992), pp. 496-517.

Patterson, Lindsay \& Brown, Alice (eds), New Scotland, New Politics? (Édimbourg, Polygon at Edinburgh, 2001).

Watson, Mike, Year Zero. An Inside View of the Scottish Parliament (Édimbourg, Polygon at Edinburgh, 2001).

Wilson, John \& Stapleton, Karyn (eds), Devolution and Identity (Aldershot, Ashgate, 2006).

\section{NOTES}

1. The Representation of the People Act, 1918. The Eligibility of Women Act, 1918. La première utilisation officielle du terme "Parlement» remonte à 1236 et le Parlement de Simon de Montfort en 1265 est considéré comme l'ancêtre du Parlement moderne, <www.parliament.uk/ about/living-heritage/evolutionofparliament/originsofparliament/birthofparliament/overview/ firstparliaments>, consulté le 25/04/2014.

2. Scotland Act 1998, premières élections à Holyrood en 1999.

3. "I propose to divide citizenship into three parts [...] civil, political and social [...] By the political element I mean the right to participate in the exercise of political power, as a member of a body invested with political authority or as an elector of the members of such a body", T. H. Marshall, Citizenship and Social Class and Other Essays (Cambridge, Cambridge University Press, 1950). Ses essais ont fait l'objet de critiques, notamment parce qu'il ne prend en compte que les hommes.

4. Dominique Schnapper, Qu'est-ce que la citoyenneté (Paris, Gallimard, 2000), p. 10.

5. "Constitutions have for the most part been constructed by men and have reflected (dominant) male perspectives on power, politics and citizenship", Carmel Roulston, "Engendering Devolution" dans John Wilson \& Karyn Stapleton (eds), Devolution and Identity (Aldershot, Ashgate, 2006), p. 143.

6. "a major aim in the establishment of the Parliament was that it should be distinct from Westminster", Mike Watson, Year Zero. An Inside View of the Scottish Parliament (Édimbourg, Polygon at Edinburgh, 2001), p. 13.

7. Groupe créé en novembre 1997 par le Secrétaire d'État à l'Écosse, qui réunissait des membres des principaux partis politiques écossais ainsi que des représentants de la société civile, et dont la mission était d'établir les bases de fonctionnement du nouveau Parlement.

8. Joni Lovenduski (ed.), State Feminism and Political Representation (Cambridge, Cambridge University Press, 2005), p. 234. Sandra Grey \& Marian Sawer (eds), Women's Movements. Flourishing or in Abeyance? (Londres, Routledge, 2008), pp. XV-XVI.

9. Christian Auer, Armel Dubois-Nayt \& Nathalie Duclos, Femmes, pouvoir et nation en Écosse du XVI siècle à aujourd'hui (Villeneuve d'Ascq, Presses du Septentrion, 2012), p. 103.

10. Véronique Molinari, «La campagne dans la campagne : pour la parité au sein des nouvelles assemblées autonomes", Revue française de civilisation britannique 14:1 ("La dévolution des pouvoirs à l'Écosse et au Pays de Galles ») (2006), p. 168.

11. Sarah Childs, Women and British Party Politics: Descriptive, Substantive and Symbolic Representation (Londres, Taylor and Francis, 2008), p. 76. Les femmes sont près de deux fois plus nombreuses dans les pays qui utilisent un scrutin proportionnel que dans ceux avec un scrutin majoritaire (15,4 \% contre 8,5 \%), Joni Lovenduski, Feminizing Politics (Cambridge, Polity Press, 2005), p. 98. 
12. Sarah Childs, Joni Lovenduski \& Rosie Campbell, Women at the Top 2005. Changing Numbers, Changing Politics? (Londres, Hansard Society, 2005), pp. 20-25.

13. <www.engender.org.uk/our-work/power-and-participation>, p. 8, consulté le 25/04/2014.

14. Ce n'est qu'à partir de 2007 que la tendance commence à diminuer pour s'inverser en 2011 : $27 \%$ des députées élues dans les circonscriptions, contre $45 \%$ des élues régionales, en raison notamment des changements de stratégies électorales des partis, "Women's Representation in Scotland and Wales", Electoral Reform Society, May 2011, <www.electoral-reform.org.uk/ downloadfile.php>, consulté le 28/04/2014.

15. Paul Chaney, "Devolution, Citizenship and Women's Political Representation in Wales" dans Esther Breitenbach \& Fiona Mackay, Women and Contemporary Scottish Politics. An Anthology (Édimbourg, Polygon at Edinburgh, 2001), p. 192.

16. Karen Bird, "The Political Representation of Women and Ethnic Minorities in Established Democracies: A Framework for Comparative Research", Working Paper presented for the Academy of Migration Studies in Denmark (AMID), Aalborg University, 11/11/2003, p. 26.

17. "Equality guarantee", "equality rhetoric", "equality promotion", Joni Lovenduski, Feminizing Politics, op. cit., p. 146.

18. Précédemment, Wendy Alexander avait dirigé les travaillistes (2007-2008) et Annabel Goldie les conservateurs (2005-2011).

19. L'ensemble des questions posées par les femmes MSPs à Holyrood (écrites et orales), mai 2011-décembre 2012, l'ensemble des contributions des femmes MPs à Westminster (écrites et orales), mai 2010-décembre 2011. Official Report, Scottish Parliament, <www.scottish.parliament.uk/parliamentarybusiness/OfficialReport.aspx>,

Hansard Parliamentary Archives, <www.publications.parliament.uk/pa/cm/cmhansrd.html>.

20. Donald Macintyre, “Commons Speaker John Bercow attacks 'sexist, snobbish, yobbish' PMQs and says he favours radical reform", The Independent, 18/02/2014, <www.independent.co.uk/ news/uk/politics/exclusive-speaker-john-bercow-attacks-sexist-snobbish-yobbish-pmqs-andsays-he-favours-radical-reform-9134744.html>, consulté le 28/04/2014.

21. "this was an aspect that had not been recommended by the CSG on the ground that it might encourage the most negative aspects associated with PMQT at Westminster", Gerry Hassan \& Chris Warhurst (eds), The New Scottish Politics, the First Year of the Scottish Parliament and Beyond (Londres, Stationary Office, 2000), p. 52.

22. John Major, The Autobiography (Londres, HarperCollins, 1999), p. 349.

23. <www.scottish.parliament.uk/visitandlearn/25488.aspx>, consulté le 25/04/2014.

24.

Drude Dahlerup, "From a Small to a Large Minority: Women in Scandinavian Politics", Scandinavian Political Studies 11:4 (1988), pp. 275-298. Le concept a, depuis, été remis en cause, notamment par son auteur même.

25. Pippa Norris \& Joni Lovenduski, Political Recruitment. Gender, Race and Class in the British Parliament (Cambridge, Cambridge University Press, 1995). Pippa Norris \& Joni Lovenduski, "Blair's Babes: Critical Mass Theory, Gender, and Legislative Life", John F. Kennedy School of Government, Harvard University, 2001. Sarah Childs, "In their Own Words: New Labour Women and the Substantive Representation of Women", British Journal of Politics and International Relations 3:2 (2001). Joni Lovenduski, Margaret Moran \& Bonnie Sones, Women in Parliament. The New Suffragettes (Londres, Politicos, 2005). Karen Bird, "Gendering Parliamentary Questions", British Journal of Politics and International Relations 7:3 (2005). Sarah Childs \& Julie Withey, "Women Representatives Acting for Women: Sex and the Signing of Early Day Motions in the 1997 Parliament", Political Studies 52:3 (2004). Sarah Childs, "Competing Conceptions of Representation and the Passage of the Sex Discrimination (Election Candidates) Bill", Journal of Legislative Studies 8:3 (2002). Rosie Campbell, Joni Lovenduski \& Sarah Childs, "Do Women Need Women MPs? A Comparison of Mass and Elite Attitudes", British Journal of Political Science 40:1 (2010). 
26. Michael J. Keating, A Test of Integration: the Scottish Members of Parliament (Glasgow, Centre for the Study of Public Policy, University of Strathclyde, 1977), p. 16, p. 31.

27. Paul Chaney, "Critical Mass, Deliberation and the Substantive Representation of Women: Evidence from the UK's Devolution Programme”, 2006, p. 35. Paul Chaney dans Esther Breitenbach \& Fiona Mackay, op. cit., pp. 189-208. Fiona Mackay, "The Impact of Devolution on Women's Citizenship in Scotland", 2006, paper presented at the 20th Congress of the International Political Science Association, Fukuoka, Japan, 2006, p. 67. Sylvia Shaw, "Gender and Politics in the Devolved Assemblies", Soundings 55, <www.newleftproject.org/index.php/site/ article_comments/gender_and_politics_in_the_devolved_assemblies>, consulté le 06/06/2014.

28. Joni Lovenduski \& Azza Karam, Women in Parliament: Making a Difference, p. 197 dans Julie Ballington \& Azza Karam (eds), Women in Parliament: Beyond Numbers (Stockholm, International Institute for Democracy and Electoral Assistance, 2005). Linda Trimble \& Jane Arscott, Still Counting: Women in Politics Across Canada (Peterborough, Broadview Press, 2003), p. 185.

29. <www.scottish.parliament.uk/parliamentarybusiness/Bills/12424.aspx\#56>, consulté le 9/05/2014.

30. Paul Chaney, "Critical Mass, Deliberation and the Substantive Representation of Women: Evidence from the UK's Devolution Programme", 2006, op. cit. Suzanne Dovi, "Will Just Any Woman, Black or Latino, Do?", American Political Science Review 96:4 (2002), pp. 729-743.

31. "key individuals that intervene repeatedly to advance feminist and gender equality concerns", Paul Chaney, 2006, op. cit., p. 22.

32. L'analyse qualitative menée ici mériterait d'être complétée par l'étude systématique de la « direction » des interventions de ces femmes, pour reprendre le terme de Beth Reingold, à savoir la distinction entre les types de contenus : féministes, anti-féministes et neutres. Beith Reingold, Representing Women (Chapel Hill, University of North Carolina Press, 2000).

33. Christian Auer, Armel Dubois-Nayt \& Nathalie Duclos, op. cit., p. 130.

34. Anne Phillips, The Politics of Presence (Oxford, Clarendon, 1995).

35. Joyce McMillan \& Ruth Fox, Has Devolution Delivered for Women? (Hansard Society and British Council Scotland, 2010), p. 10.

\section{RÉSUMÉS}

Cet article se propose d'étudier la notion de citoyenneté au prisme du processus de dévolution et de son impact sur la représentation et la participation politiques des femmes au Parlement écossais, qui a, dès sa création, assuré un meilleur équilibre que le Parlement de Westminster entre les représentants des deux sexes. L'article a pour objectif d'évaluer l'action législative des femmes députées, tant au niveau de l'investissement quantitatif dans les activités parlementaires, que de l'intérêt témoigné pour les questions traditionnellement qualifiées d'« enjeux féminins». L'approche comparatiste adoptée permet de mettre en parallèle le comportement des élues au Parlement écossais avec celui de leurs consœurs à Westminster, et, au sein de ce dernier groupe, avec celui des représentantes des circonscriptions écossaises, afin d'identifier d'éventuelles spécificités du Parlement écossais et de conclure à l'existence, ou non, d'une corrélation entre la représentation descriptive et la représentation substantive des femmes écossaises lors des dix-huit premiers mois des législatures en place. 
This paper intends to analyse the notion of citizenship through the devolution process and its impact on the political representation and participation of women in the Scottish Parliament, which has from the beginning ensured a better representation of women politicians. The paper studies the legislative action of women representatives, focusing on their quantitative involvement in various legislative activities as well as on their commitment to "women's issues". The comparative approach - women MSP, women MPs and women MPs representing Scottish constituencies - enables to identify the specificities of the Scottish Parliament and to conclude on the potential correlation between the descriptive and the substantive representation of women in Scotland over the first eighteen months of the current legislatures.

\section{INDEX}

Mots-clés : Écosse, décentralisation, représentation politique, femmes politiques

Keywords : Scotland, devolution, political representation, women politicians

\section{AUTEUR}

\section{KARINE RIVIÈRE-DE FRANCO}

CEPOC (Centre d'Études Politiques Contemporaines), composante de POLEN (EA 4710), Université d'Orléans 